\title{
Implementing and scaling-up Stepping Stones in KwaZulu-Natal, South Africa: Lessons from working in informal settlements
}

\author{
Cecilia Milford
}

M. Mtshali

Mags Beksinska

Julie Pulerwitz

Population Council

Follow this and additional works at: https://knowledgecommons.popcouncil.org/departments_sbsr-hiv

Part of the International Public Health Commons, and the Social and Behavioral Sciences Commons How does access to this work benefit you? Let us know!

\section{Recommended Citation}

Milford, Cecilia, M. Mtshali, Mags Beksinska, and Julie Pulerwitz. 2019. "Implementing and scaling-up Stepping Stones in KwaZulu-Natal, South Africa: Lessons from working in informal settlements," Results brief. Washington, DC: Population Council. 


\section{Implementing and Scaling-up Stepping Stones in KwaZulu-Natal, South Africa: lessons from working in informal settlements}

KwaZulu-Natal (KZN), a major epicentre of the South African HIV epidemic, has large informal settlements where thousands of people live. Since the settlements are unplanned, they are often underserved by municipal services and lack accessible healthcare. Given high rates of HIV, there is a critical need to assess the successes and challenges of implementing HIV prevention and treatment in this context.

Stepping Stones is a widely used life skills training intervention focused on curbing gender-based violence (GBV) and reducing HIV risk. ${ }^{1}$ The program addresses issues such as communication about HIV, relationship skills, and assertiveness. The manualbased curriculum encourages participants to engage in critical reflection through role-playing and group dialogue. Previous evaluations of Stepping Stones in South Africa found significant reductions in intimate partner violence and herpes simplex virus-2, and improvements in couple communication and negotiation, among other positive outcomes. ${ }^{2,3}$

Under Project SOAR, the Population Council and MRU (MatCH Research Unit) of the University of the Witwatersrand conducted an evaluation of the Asibonisane Community Responses (CR) program. This multi-faceted HIV and violence prevention program includes a 10-session version of Stepping Stones that was locally adapted by the Medical Research Council. CR is supported by the U.S. President's Emergency Plan for AIDS Relief (PEPFAR)/U.S. Agency for International Development (USAID) and implemented by Centre for Communication Impact (CCl) and partners. We evaluated the program in 18 selected informal settlements in the eThekwini and Ugu Districts in $\mathrm{KZN}$-one of four provinces where the program was implemented.

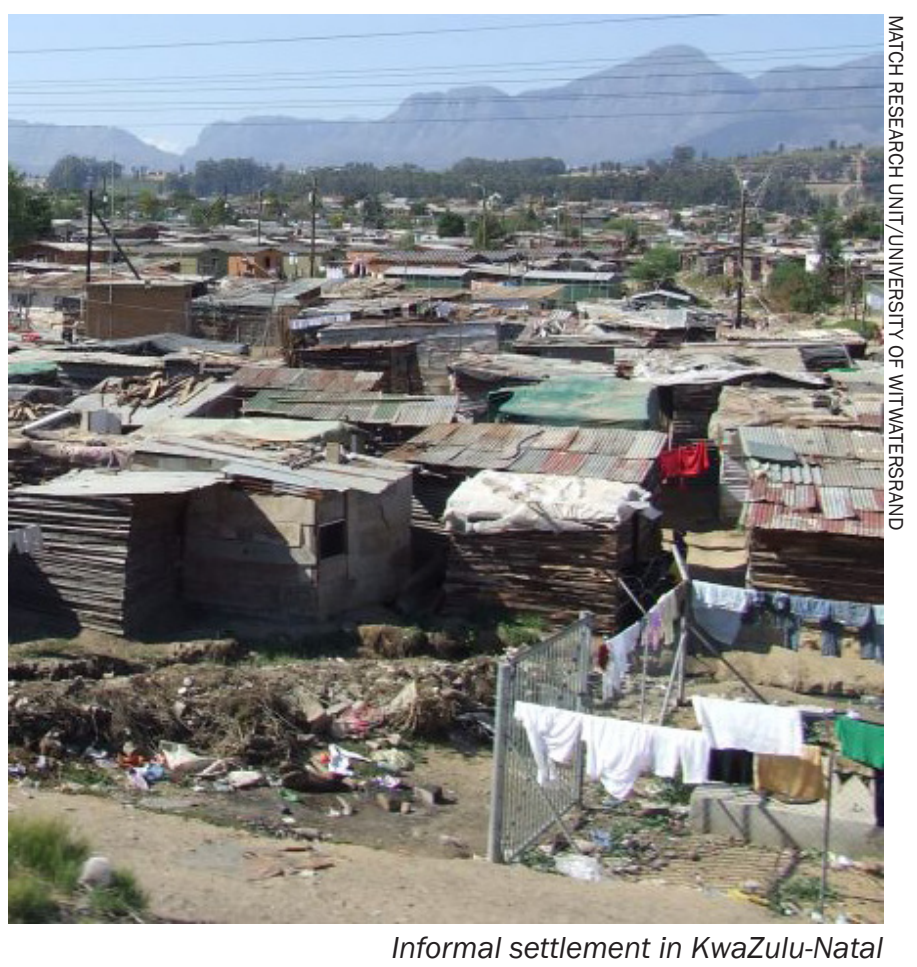

This brief focuses on the experiences, challenges, successes, and perceived effects of implementing (and scaling up) Stepping Stones in the informal settlement setting. To assess lessons learned, we conducted six focus group discussions (FGDs) and 14 in-depth interviews (IDIs) with a total of 66 community informants (34 adult males and 32 adult females) who had participated in the program. We also observed eight program sessions and conducted IDIs with 10 program facilitators/staff. 


\section{RESULTS}

Stepping Stones offered a safe and supportive learning environment.

Community members enjoyed participating in Stepping Stones because they gained knowledge and understanding, especially about HIV and AIDS and gender-related issues. Information on the biological mechanisms of HIV, caring for people living with HIV in a non-discriminatory way, and HIV prevention details, were highlighted as beneficial.

4 I liked talking about HIV. I also like talking about the immune system, what the immune system is because we were confusing these things, some would say it is this and some would say it is that. So, I learnt about them and now I know.

-Female community informant, Mixed FGD

[T]hey told us how we should treat

someone who has HIV and we should not judge him because they are the same as us.

-Female community informant, IDI

Many female participants spontaneously described the sessions on GBV as having the most appeal. They indicated that GBV was common, and felt the sessions were useful for self-protection and to access help. Many male participants also described how the sessions on GBV were useful, and that they learned that violence was wrong.

Participants (especially female participants) also described how the group environment was a safe, supportive space to discuss sensitive issues.

I was scared to talk about my problems to other people, but with Stepping Stones, I was able to release everything that was bothering me.

-Female community informant, FGD

\section{Participation was perceived to catalyze behavior change.}

Many male and about half the female community members reported that they had improved communication with their partners since participating in the Stepping Stones program.

Male community members described how learning to communicate had facilitated personal behavior change-they no longer responded violently toward their female partners. A few female participants also described how they had learned to protect themselves by becoming more independent.

1 We have communication now, like, I used to hide some things from him, but now I am able to tell him.

-Female community informant, IDI

\section{We would argue, and I would end up hitting her, but now I am able to talk} to her when we disagree on things and calm myself down to avoid hitting her.

-Male community informant, Mixed FGD

Community members (largely males) also reported improved HIV prevention behavior as a result of Stepping Stones. Some decreased their number of partners and increased condom use. The sessions on HIV also provided information on referrals which facilitated health-seeking behavior, including HIV testing and treatment, and circumcision.

had a lot of people I was with (multiple partners); I was able to get rid of some of them.

-Male community informant, IDI

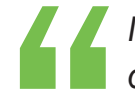

now test every month and I use a condom.

-Male community informant, IDI

Because after the program, I did not have a problem checking for HIV, you see?

-Female community informant, IDI

2 | Implementing and scaling-up Stepping Stones in KZN, South Africa: lessons from working in informal settlements 
I have referred a few, quite a few

[for circumcision]...and once he has

circumcised he will tell you that 'I have finally

done it and I am alright.'

- Staff, IDI

\section{Implementing the program in informal settlements was challenging.}

Observations and interviews with staff and participants demonstrated that finding an

appropriate venue was difficult. Some groups were conducted in informal houses or churches, and some were conducted in yards; outdoor sessions could be compromised in bad weather. While some venues appeared adequate, other venues were noisy and lacked privacy.

People look at us. Everyone who goes past the place, watches us.

-Male community informant, FGD

Observations of Stepping Stones sessions showed that activities and exercises in the manual were often not followed. Discussions with staff highlighted some of the structural challenges that led to thisincluding the limited time to conduct the sessions due to a lack of punctuality (by both facilitators and participants), competing priorities (including work and household chores), and the lack of space to conduct some activities.

The guide and the place where it needs to be implemented is not the same.

You will say that people must do role play and there is no space.... You cannot get a hall kilometers away.

\section{-Staff IDI}

During program implementation, the number of participants per group increased from the recommended 20 individuals or so to up to 40 . In the observed sessions there were large numbers of participants present.

When they are as many as 40 , they end up uncontrollable, you see. I think 20 is enough. So that everyone can understand well and if we are sitting in a circle you are able to have eye contact, but when they are a lot of them, you cannot see them properly and you forget others' names.

-Staff, IDI

\section{Well prepared facilitators were key for ensuring well run and cohesive groups.}

Most community members had confidence in their facilitators and felt that they were knowledgeable and well prepared for their group sessions. The majority were perceived as skilled and effectively able to manage group discussions, debates, or disagreements.

She was always alright. Maybe they had their problems that they were carrying, but they would put them inside and teach us, you see?

-Male community informant, IDI

However, a few community members, described lack of interest or conflict between group facilitators.

[S]ometimes you find that they would
be teaching us, but they have different
knowledge or opinion. ...And now you find
them arguing and you could see that they are
not sure of what they are teaching us about,
you see?

-Female community informant, IDI

\section{Mixed sex groups worked well.}

Many Stepping Stones sessions were mixed sex, although there were often more female than male participants in these groups. Staff and community members felt that mixed sex groups were preferable to facilitate discussions about sensitive issues.

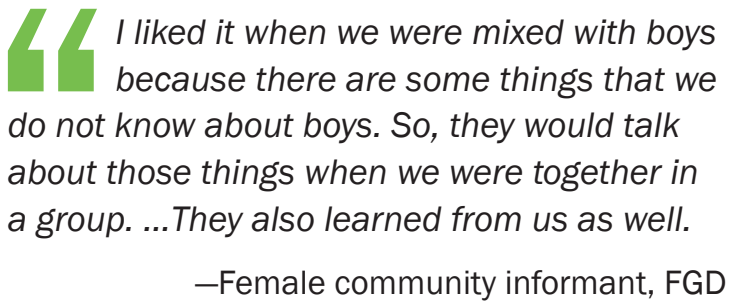

However, a few participants felt that some topics were not appropriate for both sexes (such as circumcision, condoms, and menstruation), and this was also noted during observations. 


\section{Community members suggested adding content on substance abuse and child abuse.}

Some female participants noted that child abuse and rape are common in their communities and expressed that they would have liked to have learned more about how to deal with this. Some men would have liked more information on how to deal with substance abuse, including referrals for assistance.

I do not know how I can put this, but a topic that deals with relationships with children, children being raped.... For the women with secrets.

\section{-Female community informant, IDI}

For those who are really addicted to drugs.... where can they get help to decrease the intake. Because there are people we know, who would say he is tired of smoking.

-Male community informant, IDI

\section{CONCLUSIONS AND RECOMMENDATIONS}

The CR program was well received by community members, and similar to previous research on Stepping Stones, important areas of behavior change were reported. This study also highlights difficulties in implementing Stepping Stones in the informal settlement setting. Recommendations for future implementation include:

- Prioritize indoor venues with adequate space that offer privacy and seating in order to perform group work/role plays.

- Modify session times (e.g., add evenings, weekends) to accommodate employed and student participants. Catch-up sessions should be provided for those who miss sessions.
- Shortening individual sessions may be necessary3-hour sessions are quite long where people have competing commitments such as work and household chores.

- Continue forming mixed session groups but attempt to ensure that both sexes are well represented.

- Provide facilitators with ongoing mentoring and support to address concerns and issues as they arise.

- Consider including additional topics that are relevant to the communities, e.g., child abuse, substance abuse.

\section{REFERENCES}

${ }^{1}$ MRC South Africa. 2010. Stepping Stones: A training manual for sexual and reproductive health communication and relationship skills, edition III. http://www.mrc. ac.za/sites/default/files/attachments/2016-07-08/ stepping3rdedition2010.pdf

${ }^{2}$ Jewkes, R. et al. 2008. “Impact of Stepping Stones on incidence of HIV and HSV-2 and sexual behaviour in rural South Africa: cluster randomised controlled trial," British Medical Journal 337: a506. doi: 10.1136/bmj.a506

3Jewkes, R. et al. 2014. "Stepping Stones and Creating Futures intervention: shortened interrupted time series evaluation of a behavioural and structural health promotion and violence prevention intervention for young people in informal settlements in Durban, South Africa," BMC Public Health 14: 1325. doi: 10.1186/1471-2458-14-1325

Suggested citation: Milford, C., M. Mtshali, M. Beksinska, and J. Pulerwitz. 2019. "Implementing and scaling-up Stepping Stones in KwaZulu-Natal, South Africa: lessons from working in informal settlements," Project SOAR Results Brief. Washington, DC: Population Council
Project SOAR is a six-year (September 2014-September 2020) cooperative agreement funded by the U. S. President's Emergency Plan for AIDS Relief and the U. S. Agency for International Development (Agreement No. AIDOAA-A-14-00060). The contents of this brief are the sole responsibility of Project SOAR and Population Council and do not necessarily reflect the views of PEPFAR, USAID, or the United States Government.

Population Council leads the Project SOAR consortium in collaboration with Avenir Health, Elizabeth Glaser Pediatric AIDS Foundation, the Johns Hopkins University, Palladium, and The University of North Carolina at Chapel Hill.
Project SOAR/Population Council 4301 Connecticut Avenue, NW, Suite 280 Washington, DC 20008

Tel: +1202 2379400

e-mail: ProjectSOAR@popcouncil.org projsoar.org

()Population Council, October 2019 